Within this concordant pair, both women reported engaging in oral sex and sharing wet towels during sexual activity. One woman in this pair reported recent sex with a male partner while the other woman denied history of other sexual partners during the past 3 months and had not had sex with a male partner in 5 years. Additionally, a follow-up visit of one of the members of this concordant union demonstrated a RAPD pattern discordant with previous findings indicating that the individual's initial treatment was successful and that she had acquired a new TV infection.

Conclusions Given the phenotypic similarity of banding patterns within one AAWSW sexual partnership, female to female transmission of TV may have occurred. The frequency of TV transmission between WSW is unknown at this time; however, the use of RAPD appears to be informative for differentiating isolates of TV. A prospective study examining the epidemiology and incidence of TV infection among WSW is necessary.

\section{P3-S7.06 VAGINAL DISCHARGE IN WOMEN LIVING WITH HIV ATTENDING AN AIDS CLINIC IN MANAUS, BRAZIL}

doi:10.1136/sextrans-2011-050108.489

${ }^{1} \mathrm{~A}$ Miranda, ${ }^{2} \mathrm{~L}$ Silva, ${ }^{2} \mathrm{~J}$ V Braga, ${ }^{2} \mathrm{R}$ Montes, ${ }^{2} \mathrm{~S}$ Talhari. ${ }^{1}$ Universidade Federal do, Espirito, Santo; ${ }^{2}$ Fundação de Medicina Tropical do, Amazonas, Manaus, Brazil

Background A vaginal discharge and/or vulvar itching and irritation usually characterise Vaginitis, and a vaginal odour might be present. The three diseases most frequently associated with vaginal discharge are BV (replacement of the normal vaginal flora by an overgrowth of anaerobic microorganisms, (Gardnerella vaginalis), trichomoniasis, and candidiasis.

Objectives To estimate the prevalence of vaginal discharge in HIV women attending the Institute of Tropical Medicine in Manaus, Amazonas, Brazil.

Methods A cross-sectional study performed among women attending the AIDS clinic from March to December 2010. They were invited to take pat in the study and answered an interview including demographic, behavioural and clinical data. They underwent in a gynaecological examination and it was collect vaginal samples for diagnosing Trichomonas vaginalis, Gardnerella vaginalis and Candida spp.

Results A total of 338 women were included in the study. Median age was 32 (IOR (IOR): 27; 38) years and median of schooling nine (IOR: 4; 11) years. Prevalence rate of vaginal discharge was $45.8 \%$ (95\% CI $40.5 \%$ to $51.1 \%$ ). Prevalence of Trichomonas vaginalis was $1.2 \%$ (95\% CI $0.5 \%$ to $2.4 \%$ ), Gardnerella vaginalis $35.8 \%$ (95\% CI $30.7 \%$ to $40.9 \%$ ) and Candidiasis $21.3 \%$ (95\% CI $16.9 \%$ to $25.7 \%$ ). Median of first sexual intercourse was 16 (IOR: 14; 17) years and $53.6 \%$ were married or reported a stable partner. Risk factors reported were: injecting drug use (1.2\%), no-injecting drugs $(15.2 \%)$, previous STI (32.4\%), commercial sex workers (16.4\%), more than one partner in the last year (12.4\%) and in life (94.7\%). Regarding clinical symptoms, 50,9\% reported chronic pelvic pain, $53.3 \%$ vaginal discharge, $47.6 \%$ vaginal itching, $22.8 \%$ dysuria and $9.5 \%$ genital bleeding. CD4 counts were more than 500 cells $/ \mathrm{mm}^{3}$ in $29.4 \%$ and viral load were $<1.00$ copies $/ \mathrm{ml}$ in $53.8 \%$. A total of $53.9 \%$ of women reporting vaginal discharge had a positive test for at least one disease. In the final model of logistic regression the only variable remained was having viral load $<1000$ copies/ml decreased the risk of vaginal discharge.

Conclusions Regardless the low cost and large availability of GRAM stain and cytological tests in health services for women with AIDS, there are difficulties which remain to identify interventions that refer to social, cultural and environmental influences on vaginal infections in this group.

\section{P3-S7.07 DETECTION OF TRICHOMONAS VAGINALIS IN HIV POSITIVE WOMEN IN PRETORIA, SOUTH AFRICA}

doi:10.1136/sextrans-2011-050108.490

${ }^{1} \mathrm{M}$ Kock, ${ }^{2}$ Rukasha, ${ }^{3} \mathrm{~A}$ Dijkmans, ${ }^{1} \mathrm{~A}$ Hoosen. ${ }^{1}$ University of Pretoria, National Health Laboratory Service, Pretoria, South Africa; ${ }^{2}$ University of Pretoria, Pretoria, South Africa; ${ }^{3}$ University of Leiden, Netherlands

Background The aim of this study was to detect Trichomonas vaginalis infection in HIV positive women receiving anti-retroviral therapy in Pretoria, South Africa.

Methods Self-collected vaginal swab specimens from 95 consecutive patients attending the anti-retroviral clinic (Tshwane District Hospital) were analysed. Trichomonas vaginalis was diagnosed by wet mount microscopy, culture using InPouch and a commercial PCR assay targeting the DNA repeat units. Trichomoniasis was diagnosed if any test was positive.

Results Five (5.3\%) of the 95 specimens were positive by wet mount microscopy, 21 (22.1\%) were culture positive and 28 (29.5\%) were detected by PCR. All culture and wet mount positive specimens were PCR positive. The sensitivity and specificity of wet mount microscopy compared to culture were $23.8 \%$ and $98.7 \%$ respectively. PCR detected seven additional positive specimens than culture. The specificity of PCR compared to culture was $100 \%$, with a sensitivity of $90.5 \%$. The prevalence of $T$ vaginalis was found to be $29.5 \%$ in this study.

Conclusions Previous studies in South Africa focused on the prevalence of trichomoniasis in pregnant women and women without HIV status and from lower socio-economic groups. This is the first report in HIV positive women receiving ARV treatment. There was a high prevalence $(29.5 \%)$ of $T$ vaginalis in this group. This is similar to that reported from Nigeria $(24.4 \%)$ and Ivory Coast $(27.0 \%)$, whilst the rate reported in Congolese (18.6\%) HIV positive women was lower. Using microscopy alone for the diagnosis of trichomoniasis as is the current practice in most laboratories in South Africa is inadequate and leads to missed infections.

\section{P3-S7.08 CLINICAL EVALUATION OF THE APTIMA ${ }^{\circledR}$ TRICHOMONAS VAGINALIS ASSAY ON THE TIGRIS ${ }^{\circledast}$ DTS $^{\circledR}$ SYSTEM IN ASYMPTOMATIC AND SYMPTOMATIC FEMALE SUBJECTS}

doi:10.1136/sextrans-2011-050108.491

${ }^{1} \mathrm{~J}$ Schwebke, ${ }^{2} \mathrm{M}$ Hobbs, ${ }^{3} \mathrm{~S}$ Taylor, ${ }^{4} \mathrm{~K}$ Chapin, ${ }^{5} \mathrm{M}$ Catania, ${ }^{6} \mathrm{~B}$ Weinbaum, ${ }^{5} \mathrm{D}$ Getman, ${ }^{6} \mathrm{C}$ Gaydos. ${ }^{1}$ University of Alabama, Birmingham, USA; ${ }^{2}$ University of North Carolina, Chapel Hill, USA; ${ }^{3}$ LSU Health Sciences Center, New Orleans, USA; ${ }^{4}$ Albert Brown Medical School, Providence, USA; ${ }^{5}$ Gen-Probe Incorporated, San Diego, USA; ${ }^{6}$ Johns Hopkins University, Baltimore, USA

Background This study evaluated the clinical performance of the APTIMA® Trichomonas vaginalis (ATV, Gen-Probe Incorporated) Assay, a nucleic acid amplification test for the diagnosis of Trichomonas vaginalis (TV) infection, in asymptomatic and symptomatic women.

Methods This prospective, multicenter clinical trial enrolled 1025 women attending US OB-GYN, adolescent, family planning, or sexually transmitted disease clinics. Four specimen types were collected from each subject: physician-collected vaginal swab, endocervical swab, ThinPrep specimen, and first-catch urine. Of three vaginal swabs collected from each subject, one was used for wet mount microscopic examination, one for culture, and one for molecular testing for TV. The order of collection for each vaginal swab sample was rotated to minimise sampling bias. Each specimen was tested by ATV assay using the automated TIGRIS DTS system. ATV assay performance in each sample type was determined by comparing ATV assay test result to the patient infected status (positive in saline wet mount and/or culture) for each sample. 